2 World Health Organisation. Global Action Plan for the prevention and control of non-communicable diseases, 2013-2020. Geneva, Switzerland, World Health Organisation, 2013. http://apps.who.int/iris/bitstream/10665/94384/ 1/9789241506236_eng.pdf Date last accessed: March 24, 2016.

3 GBD 2013 Mortality and Cause of Death Collaborators. Global, regional, and national age-sex specific all-cause and cause-specific mortality for 240 causes of death, 1990-2013: a systematic analysis for the Global Burden of Disease Study 2013. Lancet 2015; 385: 117-171.

4 Practical approach to lung health. Manual on initiating PAL implementation. WHO/HTM/TB/2008.410; WHO/ NMH/CHP/CPM/08.02. http://apps.who.int/iris/bitstream/10665/69937/1/WHO_HTM_TB_2008.410_eng.pdf Date last accessed: March 24, 2016.

5 Escamilla V, Emch M, Dandalo L, et al. Sampling at community level by using satellite imagery and geographical analysis. Bull World Health Organ 2014; 92: 690-694.

6 Chang AY, Parrales ME, Jimenez J, et al. Combining Google Earth and GIS mapping technologies in a dengue surveillance system for developing countries. Int J Health Geogr 2009; 8: 49.

7 Lozano-Fuentes S, Elizondo-Quiroga D, Arturo Farfan-Ale J, et al. Use of Google Earth to facilitate GIS-based decision support systems for arthropod-borne diseases. Adv Dis Surveill 2007; 4: 91.

8 Wampler PJ, Rediske RR, Molla AR. Using ArcMap, Google Earth, and Global Positioning Systems to select and locate random households in rural Haiti. Int J Health Geogr 2013; 12: 3.

9 Kamadjeu R. Tracking the polio virus down the Congo River: a case study on the use of Google Earth in public health planning and mapping. Int J Health Geogr 2009; 8: 4.

\title{
Somatic DICER1 mutations in adult-onset pulmonary blastoma
}

\author{
To the Editor:
}

Several rare lung tumours morphologically mimic embryonal structures of the developing human lung. Historically, these blastomatous tumours were described under the umbrella term of pulmonary blastoma. Subsequently, distinct entities were recognised, such as childhood pleuropulmonary blastoma (PPB) [1] (International Classification of Diseases for Oncology (ICD-O-3) code 8973/3). Later, adult-onset pulmonary blastoma was separated into well-differentiated fetal adenocarcinoma (WDFA) (ICD 8333/3) and pulmonary blastoma (ICD 8972/3) [2]. Pulmonary blastoma is a biphasic epithelial and mesenchymal malignancy, whereas PPB is purely sarcomatous and WDFA is characterised by a monophasic immature epithelium. Since 1988, the childhood PPB has received particular attention because of 1) its unique developmental progression from relatively indolent neonatal-onset lung cysts, to aggressive cystic-solid and solid sarcomas by age 72 months; 2) PPB's status heralding a newly recognised familial tumour predisposition syndrome and 3 ) its strong association with both germ-line and somatic DICER1 mutations, which is not only true for $\mathrm{PPB}$, but also for many other tumours in pleiotropic predisposition syndrome (now referred to as DICER1 syndrome). Until recently, neither WDFA nor pulmonary blastoma had been observed in families manifesting DICER1 syndrome [3]. However, in 2015, we identified a second somatic DICER1 RNase IIIb mutation [4] in a WDFA that arose in a 16-year-old germ-line DICER1 mutation carrier [5]. In addition to DICER1 mutations in PPB and WDFA, somatic CTNNB1 mutations (encoding $\beta$-catenin) appear to characterise WDFA and pulmonary blastoma [6], and are far less frequent in PPB [7, 8]. In contrast, TP53 mutations are found in both PPB [8] and pulmonary blastoma [9], but not WDFA [9].

Given the original pathological grouping of WDFA, PPB and pulmonary blastoma and the existence of partially-overlapping molecular abnormalities in these lesions, as described above, we questioned whether pulmonary blastoma might also be characterised by DICER1 mutations. We therefore analysed DICER1 status in one infant and two adult pulmonary blastomas by Sanger sequencing and/or targeted capture followed by next-generation sequencing.

The study was approved by the Institutional Review Board of the Faculty of Medicine of McGill University (A12-M117-11A) and was performed with full informed patient or parental consent. Tumours were reviewed by pathologists at the referring institutions and by central reference pathologists (D. Bouron-Dal Soglio and V-H. Nguyen). We obtained peripheral blood DNA from cases 1 (adult onset) and 3 (infant onset); formalin-fixed paraffin embedded (FFPE) tumour tissue from all cases; FFPE non-tumourous lung tissue from case 2 (adult onset); and snap-frozen tumour tissue from case 3.

Sanger sequencing and/or Fluidigm Access Array-based next-generation sequencing (Fluidigm, San Francisco, CA, USA) was used to screen for coding mutations and mutations located near the exon-intron 
boundaries in tumour genomic DNA (gDNA), as described previously [10]. Constitutional DNA was subsequently used to determine the germ-line or somatic origin of the identified mutations by PCR amplification of the region of interest followed by Sanger sequencing. DNA and RNA were extracted from FFPE samples as previously described [11].

We PCR amplified exon 3 of CTNNB1 using the following three primer pairs and a previously-described Touchdown PCR programme [12]: CTNNB1_1_F: 5'-ATGGAACCAGACAGAAAAGCG-3' and CTNNB1_1_R: 5'CAGGATTGCCTTTACCACTCA-3'; CTNNB1_2_F: 5'TTTGATGGAGTTGGACATGG-3' and CTNNB1_2_R: 5'CAGGACTTGGGAGGTATCCA-3'; CTNNB1_3_F: 5'TTTGATGGAGTTGGACATGG-3' and CTNNB1_3_R: 5'GAAGGACTGAGAAAATCCCTGTT-3'. Primer pairs were designed using Primer3 (http://bioinfo.ut.ee/primer3-0.4.0/) and UCSC in silico PCR software (http://genome.ucsc.edu/ cgi-bin/hgPcr?command=start) was used to ensure yield of a single product. Sequencing was performed by the McGill University and Genome Quebec Innovation Centre (MUGQIC) using conventional Sanger sequencing methods. Sequences were analysed visually using Lasergene Version 10 (DNASTAR; Madison, WI, USA).

Immunohistochemistry (IHC) for $\beta$-catenin and DICER1 was performed on deparaffinised $4-\mu \mathrm{m}$ tissue sections using a Ventana automated slide scanner (Ventana, Inc., Tucson, AZ, USA), following the manufacturer's instructions. The anti-DICER antibody ab14601 (Abcam, Cambridge, MA, USA) was used at a 1:50 dilution and the rabbit monoclonal anti- $\beta$-catenin antibody (Epitomics, Burlingame, CA, USA) was used at a 1:100 dilution.

We acquired three cases of pulmonary blastoma (figure 1a-i): The first was diagnosed following a history of dyspnoea in a 29-year-old female of Austrian, Lebanese, English and Jewish descent. The patient underwent a gross surgical resection, but within 3 weeks, recurrent disease was detected on computed tomography (CT) in the lower right anterior chest, centred in the pleural space. Eight cycles of combination chemotherapy with cisplatin, doxorubicin and cyclophosphamide was implemented. Despite treatment, on CT, her chest mass appeared bi-lobar with one region having decreased in size, while another had enlarged. No evidence of metastatic disease was detected. At the time of writing, additional surgical resection was under consideration. There is no family history of DICER1 syndrome-like features. The second pulmonary blastoma was diagnosed in a 25-year-old female from Catalonia, Spain, who presented with cough and haemoptysis [13]. Information on treatment and follow-up have been described by Bosch-BARRERA et al. [13]. The third pulmonary blastoma occurred in a 3-month-old child from the Basque region of Spain, who had tachypnoea and minor respiratory distress. The tumour was fully resected and the patient was treated with four cycles of combination chemotherapy consisting of carboplatin and etoposide phosphate, which was well-tolerated. Her family history is unremarkable.

The entire coding region of DICER1 was screened for mutations in tumour gDNA. We identified a predicted-truncating somatic DICER1 mutation (c.1668_1668delC; p.I557Sfs ${ }^{\star 5}$ ) and a typical RNase IIIb somatic hotspot DICER1 mutation (c.5125G>A; p.D1709N) in case 1 (figure 1j-1). Similarly, a nonsense somatic mutation (c.1232C $>$ A, p.S411*) and a RNase IIIb somatic hotspot mutation (c.5425G>A; p. G1809R) were identified in case 2 (figure $1 \mathrm{~m}-\mathrm{o}$ ). Screening of constitutional DNA confirmed the somatic origin of the identified mutations. No likely pathogenic DICER1 mutations were found in the third (infant) pulmonary blastoma case. We also screened the three patients' tumours for exon 3 CTNNB1 mutations and identified a somatic mutation in case 1 (c.110C>T, p.S37F) and case 2 (c.98C > G, p.S33C) (figure 11 and o). In accordance, the two adult tumours exhibited multifocal aberrant epithelial nuclear/ cytoplasmic $\beta$-catenin positivity on IHC staining (figure $1 \mathrm{~g}$ and $\mathrm{h}$ ). Aberrant cytoplasmic $\beta$-catenin positivity was also observed in mesenchymal cells of case 1 (data not shown).

Pulmonary blastoma is a rare subtype of sarcomatoid carcinoma and exhibits a biphasic histological pattern consisting of malignant mesenchyme and epithelium, which resemble fetal lung [2]. The DICER1 RNase IIIb hotspot missense mutation identified in each of the adult-onset pulmonary blastomas is typical of somatic mutations identified in other DICER1-associated tumours. Both hotspot mutations have been shown to interfere with the production by DICER1 of $5 p$ miRNAs consequent to the substitution of a key metal-ion binding amino acid within the catalytic RNase IIIb domain (in the case of p.D1709N), or close proximity to such a site (in the case of p.G1809R). Furthermore, the presence of a truncating DICER1 mutation coupled with the RNase IIIb hit supports the two-hit model of tumourigenesis observed in other DICER1-related lesions. The identification of these characteristic DICER1 mutations in two cases strongly suggests that adult-onset pulmonary blastoma is associated with somatic, but not germ-line mutations in DICER1. Some pulmonary blastomas (and WDFAs) contain morular structures consisting of squamoid nests with optically clear nuclei (figure $1 \mathrm{e}, \mathrm{g}$ and h). SEKINE et al. [6] noted that these morular formations are associated with the presence of somatic CTNNB1 mutations. Consistent with these prior observations, cases 1 and 2 contained both morules and a CTNNB1 mutation. Case 3 lacked both phenomena. The identified p.S37F and p.S33C CTNNB1 mutations have been previously reported to occur in lung cancers and result in the substitution of a serine residue at a glycogen synthase kinase (GSK)- $3 \beta$ phosphorylation 

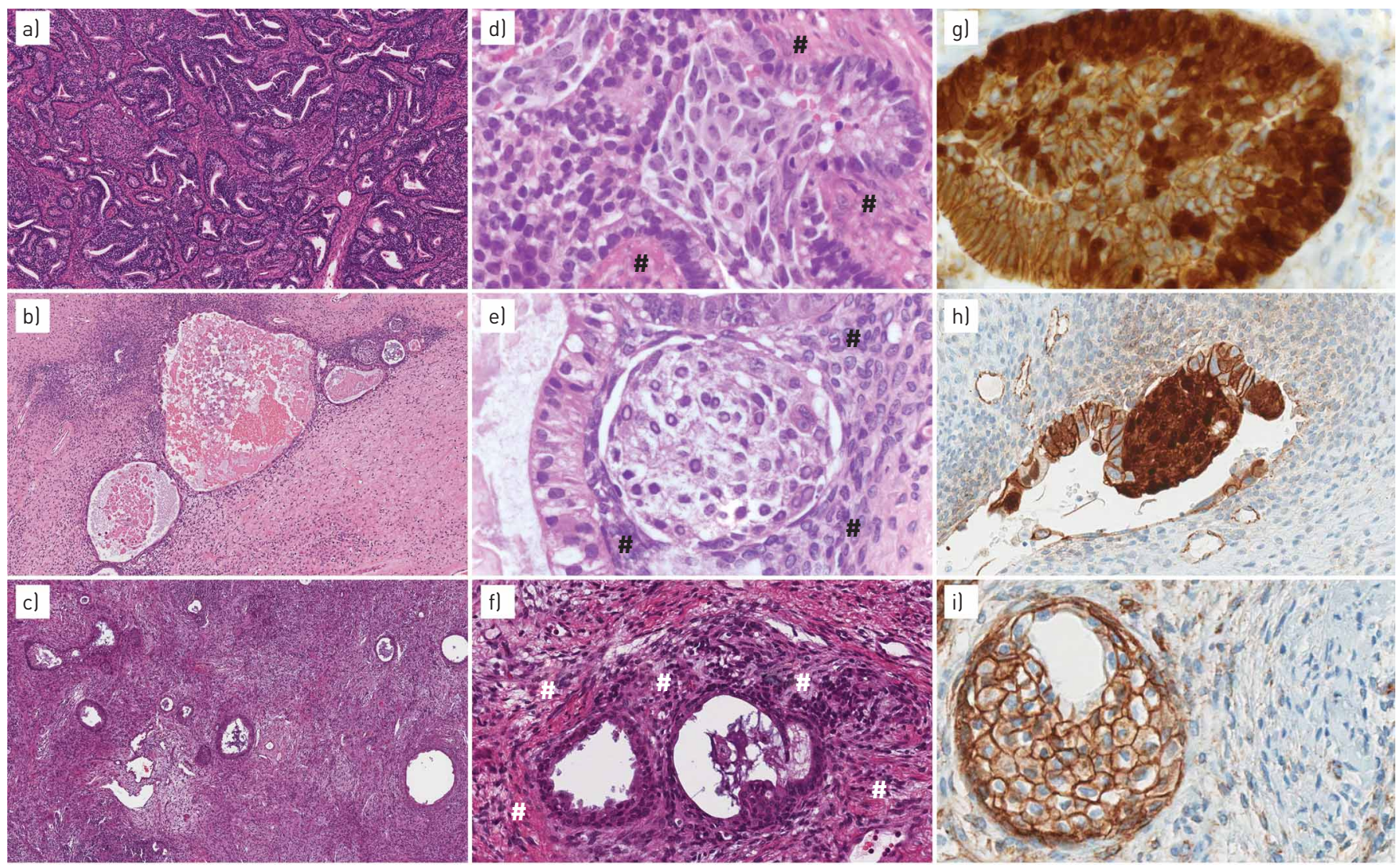

j) Truncating DICER1

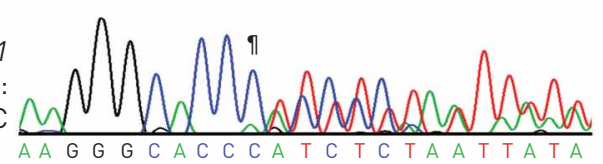

m) Nonsense DICER1

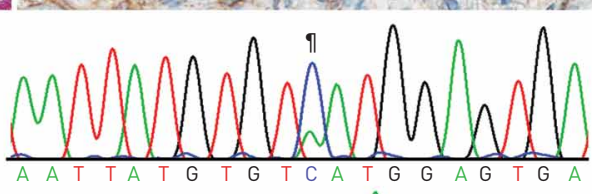

k) Missense RNase IIlb

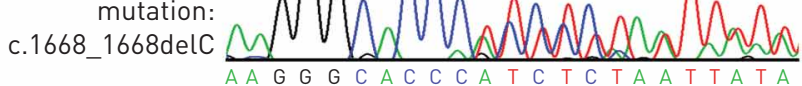

c. $1232 \mathrm{C}>\mathrm{T}$

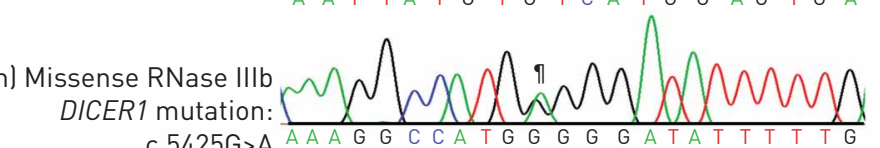
c. $5125 \mathrm{G}>\mathrm{A}$

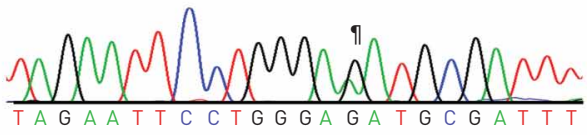
DICER1 mutation: c.5425G>A

CTNNB1 mutation:
c. $98 \mathrm{C}>\mathrm{G}$

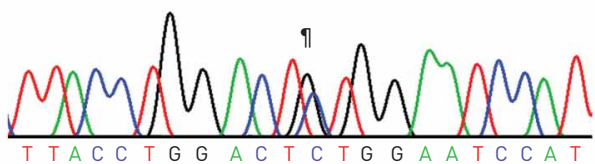

FIGURE 1 a-i) Haematoxylin and eosin staining. a-f) The epithelial glandular formations of pulmonary blastoma populate a stroma displaying variable cellularity (case 1: a and d (pre-chemotherapy); case 2: b and e (post-chemoradiotherapy); case 3: c and f (pre-chemotherapy)). Stromal compartments are shown $(\#)$ in $d$-f. $g$-i) $\beta$-catenin immunohistochemical staining: $g$ and $h$ ) The epithelium of the two adult-onset pulmonary blastomas (case $1(\mathrm{~g})$ and case 2 (h)) displayed mostly membranous, but also multifocal cytoplasmic and nuclear immunohistochemical positivity for $\beta$-catenin. h) A morular formation exhibiting a nest of epithelial cells with optically clear nuclei. i) The epithelium of the infant pulmonary blastoma (case 3) displayed membranous $\beta$-catenin positivity. j-l) Chromatograms showing somatic mutations identified in case 1 as marked (1): j) the predicted-truncating DICER1 mutation, c.1668_1668delC; k) DICER1 RNase Illb mutation, c.5125G>A; and I) the CTNNB1 c. $110 \mathrm{C}>\mathrm{T}$ mutation. $\mathrm{m}-\mathrm{0}$ ) Somatic mutations identified in case 2: $\mathrm{m}$ ) the nonsense DICER1 mutation, c.1232C $>\mathrm{T}$; n) DICER1 RNase IIlb mutation, c.5425G>A; and ol the CTNNB1 mutation, c.98C>G. The wild-type sequence is provided below each chromatogram.

site, which stabilises $\beta$-catenin and constitutively activates the WNT signalling pathway [6]. The relative importance of DICER1 mutations compared with activation of the WNT-pathway in the causation of pulmonary blastoma is unknown.

This is the first report of DICER1 mutations in pulmonary blastoma. A single WDFA has been found to harbour a germ-line DICER1 mutation [5] coupled with a somatic RNase IIIb hotspot mutation [4]. Prior to this study, PPB, pulmonary blastoma and WDFA were distinguished on morphological, immunohistochemical and molecular grounds. Our findings suggest that despite certain morphological distinctions, these diseases may have some overlapping molecular profiles that support the older pathological rubrics in which they were grouped. The similarities and differences should be explored further. New molecular data have shown that all 
three tumours possess either germ-line or somatic mutations in DICER1, and these mutations are likely to be deleterious and functionally important. We conclude that pulmonary blastoma presenting later in life can be associated with somatic DICER1 mutations. Further studies will be required to determine the generalisability of our findings, but it seems likely that DICER1 mutations will be found to be important drivers of both pulmonary blastoma and WDFA, as well as PPB. implicating DICER1 in causation http://ow.ly/10aM9V

Leanne de Kock ${ }^{1,2}$, Ismaël Bah $^{3}$, Joan Brunet ${ }^{4}$, Harriet Druker ${ }^{5}$, Itziar Astigarraga ${ }^{6,7}$, Joaquim Bosch-Barrera ${ }^{8}$, Dorothée Bouron-Dal Soglio ${ }^{9}$, Van-Hung Nguyen ${ }^{10}$, David Malkin ${ }^{11}$, John R. Priest ${ }^{12}$ and William D. Foulkes ${ }^{1,2,13,14}$ ${ }^{1}$ Dept of Human Genetics, McGill University, Montreal, QC, Canada. ${ }^{2}$ Lady Davis Institute, Segal Cancer Centre, Jewish General Hospital, Montreal, QC, Canada. ${ }^{3}$ Dept of Pathology, McGill University Health Centre, Montreal, QC, Canada. ${ }^{4}$ Dept of Medical Oncology, Hereditary Cancer Program, Catalan Institute of Oncology, Hospital Josep Trueta, Girona, Spain. ${ }^{5}$ Division of Genetic Counselling and Haematology/Oncology, The Hospital for Sick Children; Dept of Molecular Genetics, The University of Toronto, Toronto, ON, Canada. ${ }^{6}$ Servicio de Pediatria, Bio Cruces Health Research Institute, Hospital Universitario Cruces, Barakaldo, Bizkaia, Spain. ${ }^{7}$ Departamento de Pediatria, Universidad del Pais Vasco UPV/ EHU, Spain. ${ }^{8}$ Dept of Medical Oncology, Catalan Institute of Oncology, Doctor Josep Trueta University Hospital, Girona, Spain. ${ }^{9}$ Dept of Pathology, CHU-Sainte Justine and University of Montreal, Montréal, QC, Canada. ${ }^{10}$ Division of Pediatric Pathology, The Montreal Children's Hospital, McGill University Health Centre, Montreal, QC, Canada. ${ }^{11}$ Division of Haematology/Oncology, The Hospital for Sick Children; Depts of Pediatrics and Medical Biophysics, The University of Toronto, Toronto, ON, Canada. ${ }^{12}$ Minneapolis, Minnesota, USA. ${ }^{13}$ Dept of Medical Genetics, Research Institute of the McGill University Health Centre, Montreal, QC, Canada. ${ }^{14}$ Program in Cancer Genetics, Department of Oncology and Human Genetics, McGill University, Montreal, QC, Canada.

Correspondence: William D. Foulkes, Dept of Medical Genetics, Lady Davis Institute, Segal Cancer Centre, Jewish General Hospital, 3755 Cote St. Catherine Road, Montreal, QC, Canada, H3T 1E2. E-mail: william.foulkes@mcgill.ca

Received: Jan 222016 | Accepted after revision: March 102016 | First published online: April 282016

Support statement: This research was made possible thanks to the Alex's Lemonade Stand Foundation grant to William D. Foulkes and the Vanier Canada Graduate Scholarship to Leanne de Kock. Joan Brunet benefited from a Spanish Society of Medical Oncology grant. Funding information for this article has been deposited with FundRef.

Conflict of interest: Disclosures can be found alongside the online version of this article at erj.ersjournals.com

Acknowledgements: We thank Talia Boshari (Lady Davis Institute, Jewish General Hospital, Montreal, QC, Canada) for help with sample acquisition and associated administrative work and Naciba Benlimame (George and Olga Minarik Research Pathology Facility, Jewish General Hospital, Montreal, QC, Canada) for help with IHC staining. We also thank Richard Hung (St Joseph's Health Centre, Toronto, ON, Canada) for performing the initial pathology review on case 1.

\section{References}

1 Manivel JC, Priest JR, Watterson J, et al. Pleuropulmonary blastoma. The so-called pulmonary blastoma of childhood. Cancer 1988; 62: 1516-1526.

2 Koss MN, Hochholzer L, O’Leary T. Pulmonary blastomas. Cancer 1991; 67: 2368-2381.

3 Priest JR, Williams GM, Hill DA, et al. Pulmonary cysts in early childhood and the risk of malignancy. Pediatr Pulmonol 2009; 44: 14-30.

4 de Kock L, Bah I, Wu Y, et al. Germline and somatic DICER1 mutations in a well-differentiated fetal adenocarcinoma of the lung. $J$ Thorac Oncol 2016; 11: e31-e33.

$5 \mathrm{Wu} \mathrm{Y}$, Chen $\mathrm{D}, \mathrm{Li} \mathrm{Y}$, et al. DICER1 mutations in a patient with an ovarian Sertoli-Leydig tumor, well-differentiated fetal adenocarcinoma of the lung, and familial multinodular goiter. Eur J Med Genet 2014; 57: 621-625.

6 Sekine S, Shibata T, Matsuno Y, et al. Beta-catenin mutations in pulmonary blastomas: association with morule formation. J Pathol 2003; 200: 214-221.

7 Seki M, Yoshida K, Shiraishi Y, et al. Biallelic DICER1 mutations in sporadic pleuropulmonary blastoma. Cancer Res 2014; 74: 2742-2749.

8 Pugh TJ, Yu W, Yang J, et al. Exome sequencing of pleuropulmonary blastoma reveals frequent biallelic loss of TP53 and two hits in DICER1 resulting in retention of 5p-derived miRNA hairpin loop sequences. Oncogene 2014; 33: 5295-5302.

9 Bodner SM, Koss MN. Mutations in the p53 gene in pulmonary blastomas: immunohistochemical and molecular studies. Hum Pathol 1996; 27: 1117-1123.

10 de Kock L, Sabbaghian N, Plourde F, et al. Pituitary blastoma: a pathognomonic feature of germ-line DICER1 mutations. Acta Neuropathol 2014; 128: 111-122.

11 de Kock L, Sabbaghian N, Druker H, et al. Germ-line and somatic DICER1 mutations in pineoblastoma. Acta Neuropathologica 2014; 128: 583-595.

12 Witkowski L, Mattina J, Schonberger S, et al. DICER1 hotspot mutations in non-epithelial gonadal tumours. $\mathrm{Br} \mathrm{J}$ Cancer 2013; 109: 2744-2750.

13 Bosch-Barrera J, Holguin F, Baldo X, et al. Neoadjuvant chemoradiotherapy treatment for a classic biphasic pulmonary blastoma with high PD-L1 expression. Anticancer Res 2015; 35: 4871-4875. 